\title{
Halo formation due to beam-beam interactions of beams optically mismatched at injection
}

\author{
Ji Qiang \\ Lawrence Berkeley National Laboratory, Berkeley, California 94720, USA
}

(Received 13 January 2004; published 30 March 2004)

\begin{abstract}
In this paper, we report on the halo formation and emittance growth driven by a parametric resonance during beam-beam collisions with optically mismatched injection. In the regime of the weak-strong beam-beam interaction, if two beams have the same machine tunes, on-axis head-on collisions between a mismatched strong beam and a weak beam will not cause the formation of halo. However, if the two beams collide with an initial offset, the beam-beam force from the mismatched strong beam can cause halo formation and emittance growth in the weak beam. Meanwhile, if two beams have different machine tunes, for opposite charged colliding beams, when the machine tune of the weak beam is smaller than that of the strong beam, there is emittance growth in the weak beam. When the machine tune of the weak beam is larger than that of the strong beam, there is little emittance growth. In the regime of the strong-strong beam-beam interaction, halo is formed in both beams even when the two beams collide head-on on the axis with equal machine tunes. This puts a strong requirement for a good beam match during the injection to colliders in order to avoid emittance growth if there are no other mechanisms after the injection to decohere the mismatched envelope oscillation.
\end{abstract}

DOI: 10.1103/PhysRevSTAB.7.031001

\section{INTRODUCTION}

In high energy colliders, large amplitude halo particles from the tails of colliding beams cause background in experiments. They also reduce the lifetime of colliding beams and limit the luminosity that can be achieved. A large loss of particles can also destroy collimators and can quench the operation of a superconducting collider. A number of mechanisms, e.g., Arnold diffusion, resonance streaming, and modulational diffusion, that generate such halo particles have been studied [1-4] and been recently reviewed by Zimmermann [5]. These studies assumed that the beams are well matched into the colliders. When the beams are injected into colliders with an initial mismatch, another mechanism, i.e., the parametric resonance, may cause formation of halo particles and beam emittance growth. In this study, we observed halo formation and emittance growth in the weak beam when the strong beam is mismatched during the weak-strong beam-beam interaction with an initial offset and with different machine tunes. During the strong-strong beambeam interaction, emittances grow in both beams when even one of the colliding beams is mismatched.

Halo formation driven by the parametric resonance has been extensively studied in the context of high-intensity proton linacs due to its direct connection with beam losses in these machines [6-12]. High-intensity proton accelerators are required to minimize the particle loss to the wall of the beam pipe in order to reduce the risk of radioactivation. An important mechanism for beam loss is the presence of a large amplitude, low intensity beam halo far from the beam core. One potential way to generate these large amplitude halo particles is through the parametric resonance between individual particles and a mismatched envelope oscillation. The mismatched envelope oscillation occurs when the beam is injected into a
PACS numbers: 29.27.Bd, 41.75.-i

section of focusing lattice which is not well matched to the preceding one. In these studies, the nonlinear spacecharge forces from the Coulomb interactions among charged particles play an important role in halo formation. In high energy ring colliders, the nonlinear spacecharge forces within the beam itself are negligible due to the cancellation of electric forces and magnetic forces. However, the space-charge forces from the oppositely moving beam are not canceled but instead add up. If a beam is not injected into a collider with a proper match, the envelope of the beam starts to oscillate. During the beam-beam interaction, this mismatched envelope oscillation can resonate with the particles in the oppositely moving beam or with particles in the beam itself. This causes halo formation and emittance growth which can reduce the luminosity of beam-beam collisions.

The organization of this paper is as follows: The particle-core model for halo formation driven by parametric resonance is described in Sec. II. Halo formation from the mismatched weak-strong beam-beam interaction is presented in Sec. III. Halo formation from the mismatched strong-strong beam-beam interaction is given in Sec. IV. Conclusions are drawn in Sec. V.

\section{THE PARTICLE-CORE MODEL FOR HALO FORMATION}

The mechanism of halo formation driven by parametric resonance has been studied using a particle-core model in high-intensity proton $\mathrm{rf}$ linacs for a round continuous beam and for a bunched beam $[6,10,12]$. Here, we are going to use a round continuous beam approximation to the real beam in a hadron collider. In the particle-core model, the beam consists of a core and test particles. The core, which contains most particles, is modeled by the rms envelope equations. The test particles 
contain a small fraction of the beam and are subject to the effects of external focusing forces and space-charge forces from the core. The effects of test particles on the core and the mutual Coulomb interactions among test particles are neglected. For a round continuous beam, the envelope equation of rms radius $R$ can be written as

$$
\frac{d^{2} R}{d s^{2}}+k_{0}^{2} R-\frac{\epsilon^{2}}{R^{3}}+F_{\mathrm{sc}}(R)=0,
$$

where $s$ is the longitudinal coordinate, $k_{0}$ is the wave number from external focusing, $\epsilon$ is the transverse rms emittance, and $F_{\mathrm{sc}}$ is the term associated with the spacecharge force, which depends on the particle distribution inside the beam. A constant wave number $k_{0}$ is used, which corresponds to a smooth approximation to a real focusing lattice. We have also neglected the effects of dispersion in the above equation. From the envelope equation, we can find a stationary solution of the rms radius $R$ from the solution of the equation,

$$
k_{0}^{2} R-\frac{\epsilon^{2}}{R^{3}}+F_{\mathrm{sc}}(R)=0 .
$$

When a beam is injected into the accelerator with the stationary radius, the beam is well matched and the beam envelope stays constant. When the beam is injected into the accelerator with a different radius, the beam is mismatched and the beam envelope starts to oscillate. The oscillation wave number can be represented as $k_{e}$ and can be obtained from the linearized envelope equation (1).

The vertical equation of motion for a test particle subject to external focusing forces and the space-charge force of the core is

$$
\frac{d^{2} y}{d s^{2}}+k_{0}^{2} y+G(y, R, s)=0
$$

where $G(y, R, s)$ represents the space-charge force which depends on the location of the test particle, the envelope of the beam, and the particle distribution of the beam. For a mismatched beam, when the oscillation wave number of the test particle $k_{y}$ satisfies the condition $k_{y} / k_{e}=m / n$, where $m$ and $n$ are integers, the test particle is going to resonate with the oscillation of the envelope and is driven to large amplitude. This process is the so-called parametric resonance. In practical applications, the most dangerous resonance is the low order resonance $k_{y} / k_{e}=1 / 2$, i.e., the 1:2 resonance, which causes the particle to move to large amplitude and to form the halo. A typical stroboscopic plot of the 1:2 resonance in phase space for a constantly focused beam is given in Fig. 1. Here, the particle amplitude at outer edge of the 1:2 resonance can be as large as 3 times the matched beam edge radius. These large amplitude particles are halo particles and can be lost to a conducting pipe.

In high energy colliders, the space-charge forces within the bunch itself are negligible. The space-charge forces acting on the test particles are from the oppositely moving charged beam. During the beam-beam interaction, when the intensity of one beam is much larger than that of the opposite beam, this beam is regarded as a strong beam and the opposite beam is regarded as a weak beam. The beam-beam space-charge forces from the weak beam to the strong beam are neglected. Under this condition, the mismatched strong beam will oscillate with a wave number $k_{e}^{s}=2 k_{0}^{s}$. The particles in the weak beam can be regarded as test particles in the particle-core model. For an azimuthally symmetric strong beam with a centroid on the axis, the oscillation wave number of the particles in the weak beam is going to have a minimum or

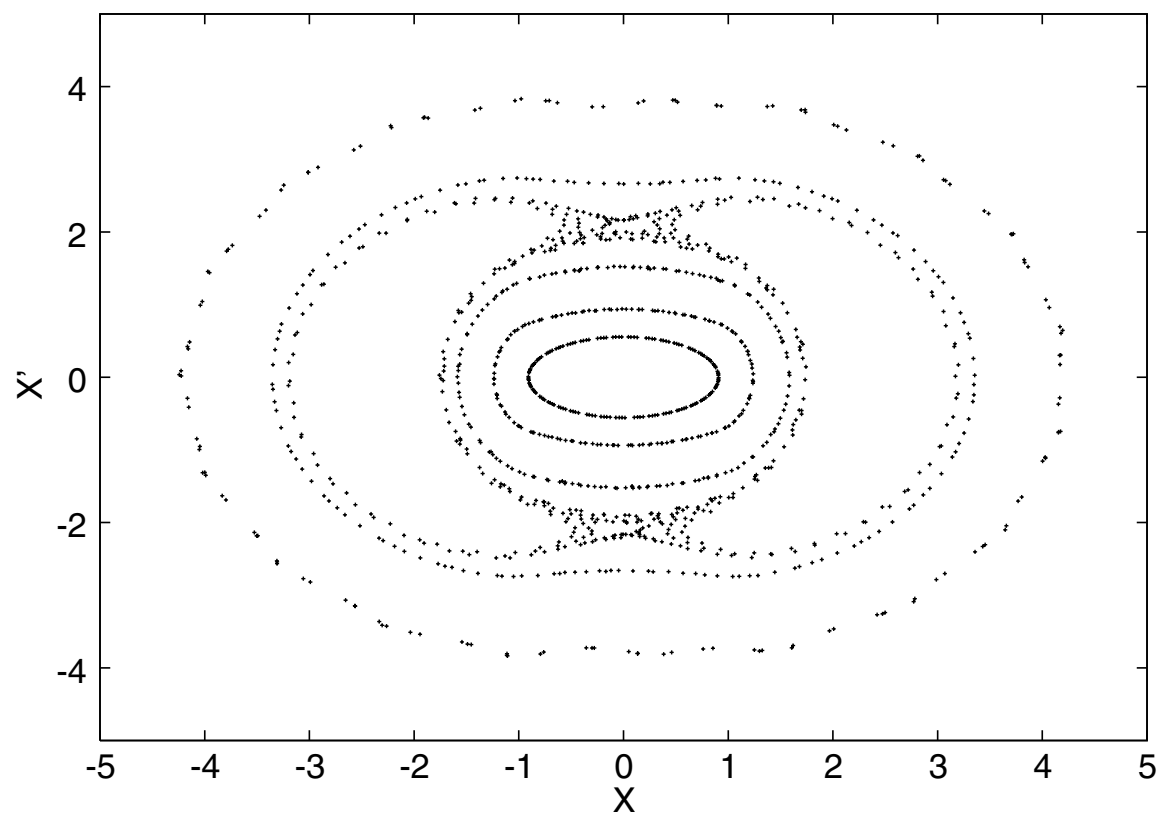

FIG. 1. A stroboscopic plot of the 1:2 resonance in the $x-x^{\prime}$ plane. 
maximum (depending on the sign of the charge) at $k_{0}^{w}$ since there is no space-charge force at infinite distance. If the strong beam and the weak beam have the same machine tunes, i.e., $k_{0}^{s}=k_{0}^{w}$, there will be no 1:2 resonance between the particles in the weak beam and the envelope oscillation of the strong beam. If $k_{0}^{s} \neq k_{0}^{w}$, there exists a potential 1:2 resonance depending on the sign of charge of two colliding beams. When the centroid of the strong beam is not on the axis, e.g., with an initial offset, the minimum or maximum wave number of the particles in the weak beam can be smaller or larger than $k_{0}^{w}$. In this case, there will be a 1:2 resonance between some particles of the weak beam and the envelope oscillation of the strong beam if both beams have the same machine tunes.

When the intensities of two colliding beams are comparable, the beam-beam space-charge forces from each beam are no longer negligible. This case is called the strong-strong beam-beam interaction. The wave number of the mismatched envelope oscillation is different from $2 k_{0}$. A test particle can resonate with the envelope oscillation even without the initial beam offset or the machine tune difference.

\section{HALO FORMATION FROM THE WEAK- STRONG BEAM-BEAM INTERACTION}

In this section, we study halo formation from a mismatched weak-strong beam-beam interaction using macroparticle simulation. Here, we have used a group of

TABLE I. The nominal parameters for the weak-strong beam-beam interaction.

\begin{tabular}{lc}
\hline \hline Beam energy $(\mathrm{GeV})$ & 150 \\
Protons per bunch & $1.7 \times 10^{11}$ \\
$\alpha$ & $(-0.116,0.054)$ \\
$\beta^{*}(\mathrm{~m})$ & $(1.56,1.76)$ \\
Unnormalized rms emittance $(\mathrm{mm} \mathrm{mrad})$ & $(0.0261,0.0261)$ \\
Betatron tunes $\left(\nu_{x}, \nu_{y}\right)$ & $(0.585,0.575)$ \\
rms bunch length $(\mathrm{m})$ & 0.37 \\
Synchrotron tune $\nu_{z}$ & 0.0007 \\
\hline \hline
\end{tabular}

parameters similar to those of the Tevatron at injection energy, where the weak-strong beam-beam interaction is a good approximation. Table I gives a list of the nominal parameters for the strong proton beam. The weak (antiproton) beam has the same parameters except for a lower intensity.

In the simulation, we have assumed a Gaussian distribution for the strong beam. The beam-beam forces from the strong beam to the weak beam are calculated using a complex error function [13]. Around 1000000 particles have been used to track the particles in the weak beam.

We first study on-axis head-on collisions between the weak antiproton beam and a 20\% mismatched strong proton beam. The envelope of the strong beam oscillates with a wave number twice that of the machine betatron tune. There is no decoherence process modeled for the strong beam envelope oscillation. Figure 2 shows the vertical normalized emittance growth of the weak beam (left panel), the tune spread of the weak beam (middle panel), and the vertical phase space distribution after 1000 turns (right panel).

We see that even with the presence of the initial envelope mismatch of the strong beam, there is little emittance growth in the weak beam. This can be understood by checking the particle tune [wave number/ $(2 \pi)$ ] spread in the weak beam. From the tune spread of the weak beam shown in the middle panel of Fig. 2, we see that the particles in the weak beam have a tune greater than the machine bare tune due to the focusing of the beam-beam forces. There is no 1:2 parametric resonance to move the particles in the weak beam to large amplitude and to form halo. The particle phase space plot in the vertical plane shows no resonance structure after 1000 turns.

In the above case, we have assumed that two beams collide on axis. In practice, the two beams may not collide exactly on axis, e.g., in the case of parasitic collisions. When the two beams collide with an offset, the beambeam forces are no longer symmetric in the vertical direction. Some particles in the weak beam can be defocused. Figure 3 shows the vertical normalized emittance growth of the weak beam (left panel), the tune spread of
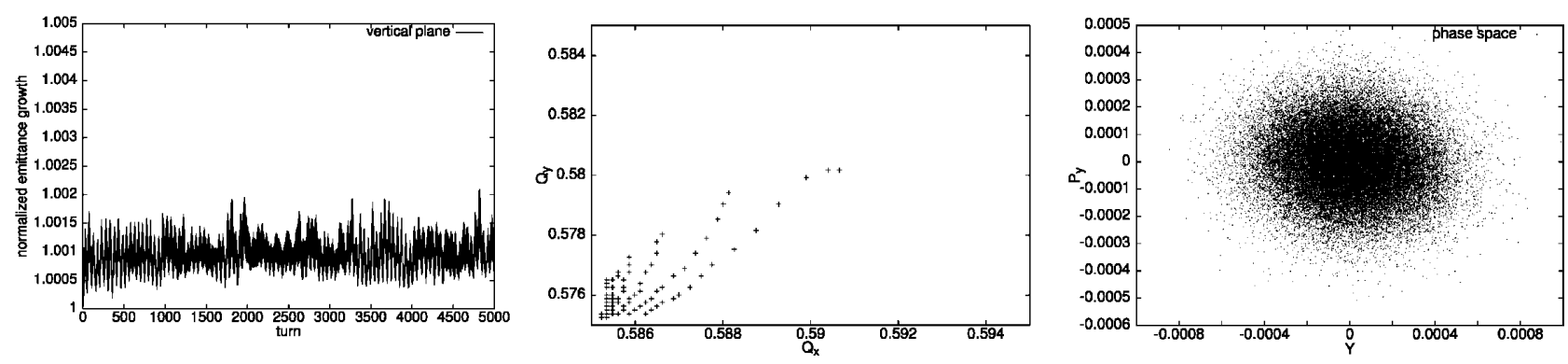

FIG. 2. The vertical normalized emittance growth of the weak beam (left panel), the tune spread of the weak beam with initial maximum $5 \sigma$ amplitude range (middle panel), and the vertical phase space distribution after 1000 turns (right panel), with an initial $20 \%$ mismatch of the strong beam and on-axis collision. 

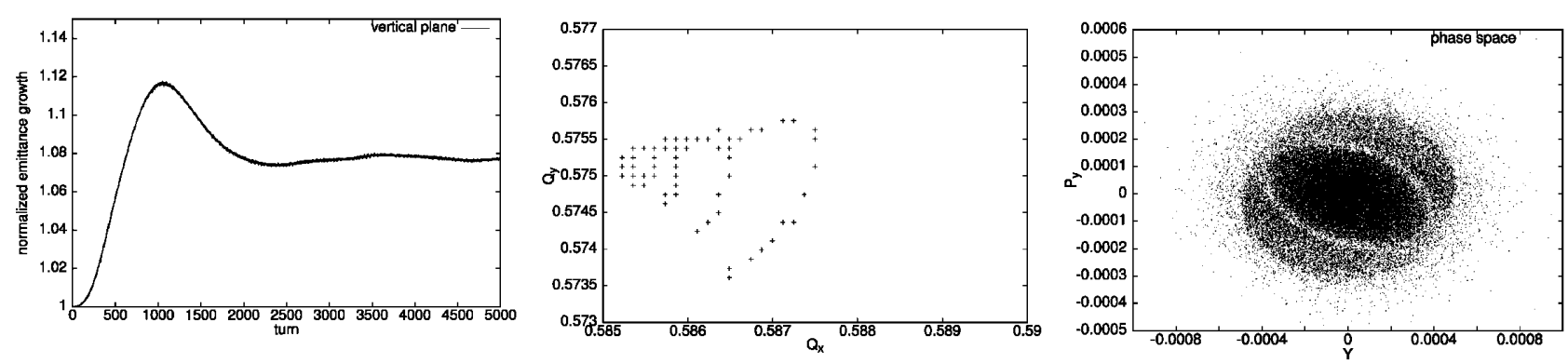

FIG. 3. The vertical normalized emittance growth of the weak beam (left panel), the tune spread of the weak beam with initial maximum $5 \sigma$ amplitude range (middle panel), and the vertical phase space distribution after 1500 turns (right panel), with an initial $20 \%$ mismatch of the strong beam and $2 \sigma$ vertical offset collision.

the weak beam (middle panel), and the vertical phase space distribution after 1500 turns (right panel). The strong beam has an initial $20 \%$ mismatch and is offset $2 \sigma$ from the axis in the vertical direction.

We see that the vertical emittance of the weak beam has increased by $8 \%$ after 2000 turns. The particle vertical tune spread in the weak beam has a range of 0.5738 to 0.5758 . This suggests that some particles should have a vertical tune $k_{y}^{w}=k_{0}^{w}=0.575$. These particles will resonate with the mismatched envelope oscillation of the strong beam, which has a wave number $2 k_{0}^{s}$, through the $1: 2$ resonance and will form the halo. From the vertical phase space plot on the right of Fig. 3, we see the 1:2 resonance structure in the weak beam after 1500 turns.

When the two beams have different machine tunes, i.e., $k_{0}^{w} \neq k_{0}^{s}$, there is also potential halo formation in the weak beam when the strong beam is initially mismatched. For a proton-antiproton collider, the beam-beam forces increase the particle tune in the weak beam. If the machine tune of the weak beam is lower than that of the strong beam, the beam-beam collision will cause an increase of the tune of individual particles in the weak beam. This causes some particles in the weak beam to resonate with the mismatched envelope oscillation of the strong beam through the 1:2 resonance and to form halo in the weak beam.

Figure 4 shows the vertical normalized emittance growth of the weak beam (left panel), the tune spread of the weak beam (middle panel), and the vertical phase space distribution after 1000 turns (right panel). The vertical machine tune of the weak beam is 0.573 which is 0.002 lower than that of the strong beam. We see that the vertical emittance has grown by $8 \%$ after 5000 turns. The particle tune spread in the weak beam shows that some particle tunes have crossed the 1:2 resonance line in the vertical direction. The resonance structure has appeared in the vertical phase space plane after 1000 turns as seen in the right plot of Fig. 4. When the machine tune of the weak beam is higher than that of the strong beam, there is no halo formed from the 1:2 resonance. This is because all particles in the weak beam have vertical tunes greater than the machine tune of the strong beam. Figure 5 shows the emittance evolution of the weak beam with initial 0.002 higher machine tune than the strong beam. There is little (only $0.2 \%$ ) emittance growth after 5000 turns.

The amount of emittance growth also depends on the extent of the mismatch of the strong beam during the weak-strong beam-beam interaction. For a larger strong beam mismatch, there is a larger emittance growth of the weak beam. Figure 6 shows a comparison of the emittance evolution with $20 \%$ and $50 \%$ initial mismatch of the strong beam.

In this case, the two beams collide head-on on the axis. The weak beam machine tune is 0.002 lower than that of the strong beam. We see that the final emittance growth has increased from $8 \%$ to $30 \%$.
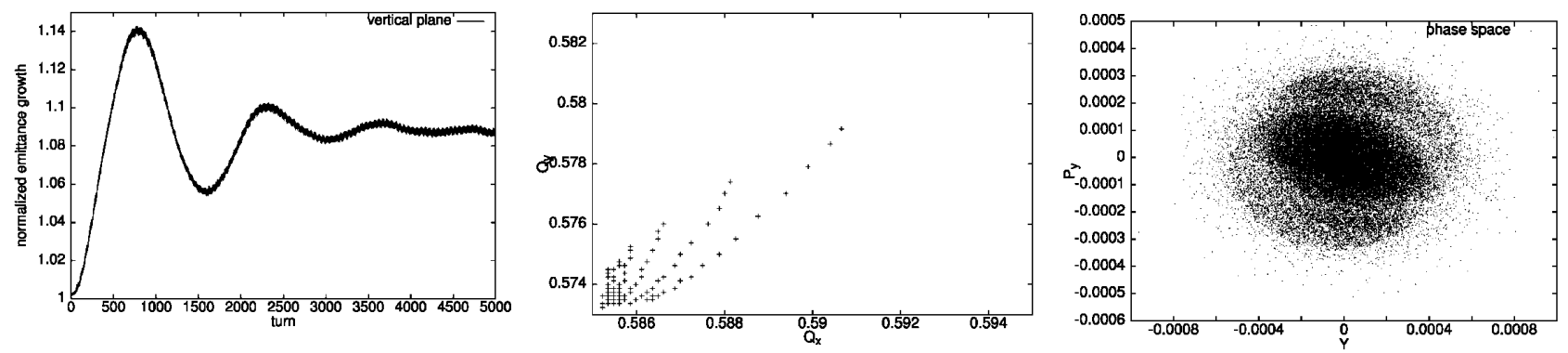

FIG. 4. The vertical normalized emittance growth of the weak beam (left panel), the tune spread of the weak beam with initial maximum $5 \sigma$ amplitude range (middle panel), and the vertical phase space distribution after 1000 turns (right panel), with an initial 20\% mismatch of the strong beam and 0.002 lower machine tune of the weak beam. 


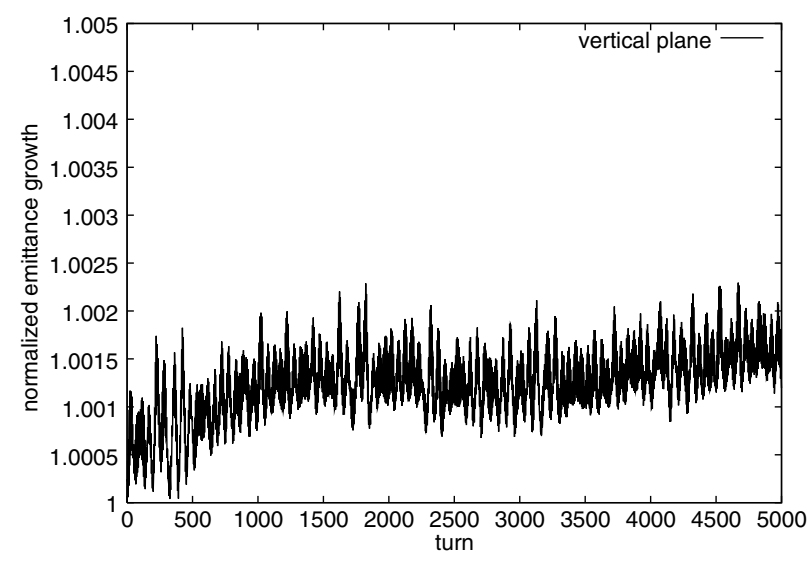

FIG. 5. The vertical normalized emittance growth of the weak beam with an initial $20 \%$ mismatch of the strong beam and 0.002 higher machine tune of the weak beam.

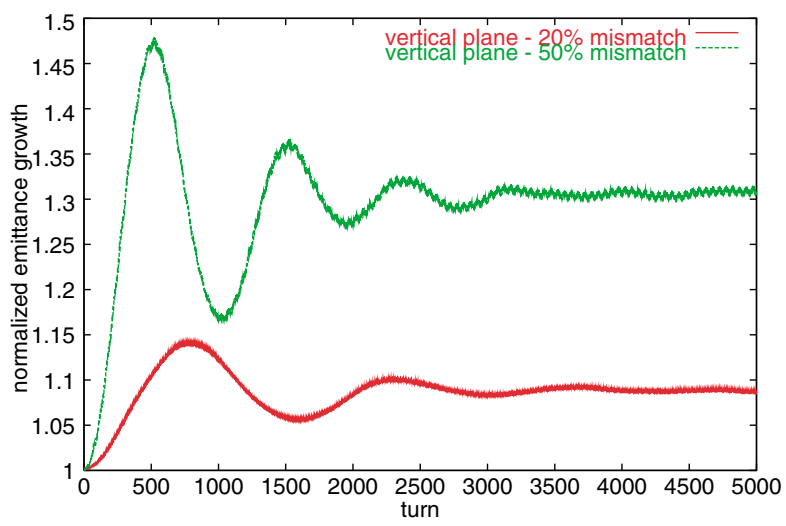

FIG. 6. (Color) The vertical normalized emittance growth of the weak beam with an initial $20 \%$ and $50 \%$ mismatch of the strong beam and 0.002 lower machine tune of the weak beam.

\section{HALO FORMATION FROM THE STRONG- STRONG BEAM-BEAM INTERACTION}

During the strong-strong beam-beam interaction, the space-charge forces from both beams are not negligible. The wave number of the mismatched envelope oscillation is modified with the presence of beam-beam forces. For two colliding beams with the same type of charge, the wave number of the mismatched envelope oscillation is reduced and is below $2 k_{0}$. This makes it possible for individual particles with tune near $k_{0}$ to resonate with the envelope oscillation through the 1:2 parametric resonance and to form halo. This parametric resonance results in emittance growth of the two colliding beams and also decoheres the mismatched envelope oscillation.

To study the halo formation from the mismatched strong-strong beam-beam interaction, we have used a group of parameters from the nominal Large Hadron Collider (LHC) design. The parameters are given in Table II. The beam-beam parameter for this design is 0.0034 .
TABLE II. LHC nominal beam-beam parameters.

\begin{tabular}{lc}
\hline \hline Beam energy $(\mathrm{TeV})$ & 7 \\
Protons per bunch & $1.05 \times 10^{11}$ \\
$\beta^{*}(\mathrm{~m})$ & 0.5 \\
rms spot size at the IP $(\mu \mathrm{m})$ & 15.9 \\
Betatron tunes $\left(\nu_{x}, \nu_{y}\right)$ & $(0.31,0.32)$ \\
rms bunch length $(\mathrm{m})$ & 0.077 \\
Synchrotron tune $\nu_{z}$ & 0.0021 \\
\hline \hline
\end{tabular}

The simulations have been done using a recently developed parallel strong-strong/strong-weak beam-beam code with $1 \times 10^{6}$ macroparticles and $128 \times 128$ numerical grid [14]. In the simulation, we have neglected the effects of finite bunch length of the beam and have used one slice for each beam. The beam-beam collision is also assumed to be a head-on collision with zero crossing angle. Figure 7 shows the emittance evolution without and with initial $20 \%$ mismatch in both planes of one beam.

It is seen that without initial mismatch, there is little emittance growth in the horizontal and vertical planes for both beams. With $20 \%$ initial mismatch in the first beam, several percent emittance growth is observed in both beams. The emittance growth in both beams can be understood from the power spectrum of the mismatched envelope oscillation and the spectrum of the centroid oscillations. Figure 8 shows the power spectra of the horizontal centroid motion of the two beams and the spectrum of the envelope oscillation of the mismatched beam. We see that besides the coherent $\sigma$ mode and $\pi$ mode, there is a continuum spectrum between the $\pi$ mode (0.306) and the $\sigma$ mode $(0.31)$ due to the incoherent motion of individual particles in both beams. From the right plot of Fig. 8, the envelope oscillation frequency has moved down from 0.62 to 0.618 . The range of twice the frequency of the incoherent particle motion is from 0.612 to 0.62 . This results in a 1:2 parametric resonance between some particles in both beams and the mismatched envelope oscillation, which causes the emittances to grow in both beams.

If both beams are mismatched initially, there will be more oscillatory free energy available that can cause more emittance growth. Figure 9 shows the emittance evolution in the horizontal and vertical planes of both beams with initial $20 \%$ mismatch in both beams.

Comparing with Fig. 7, we see that the total emittances (the sum of the horizontal and the vertical emittances) in both beams have been almost doubled.

In the above case, we have assumed that two strong beams have the same machine tunes and the collision is on axis. When the two beams collide with an initial offset or the machine tunes for the two beams are unequal, there is also emittance growth driven by the mismatched envelope oscillation as was observed in the mismatched 

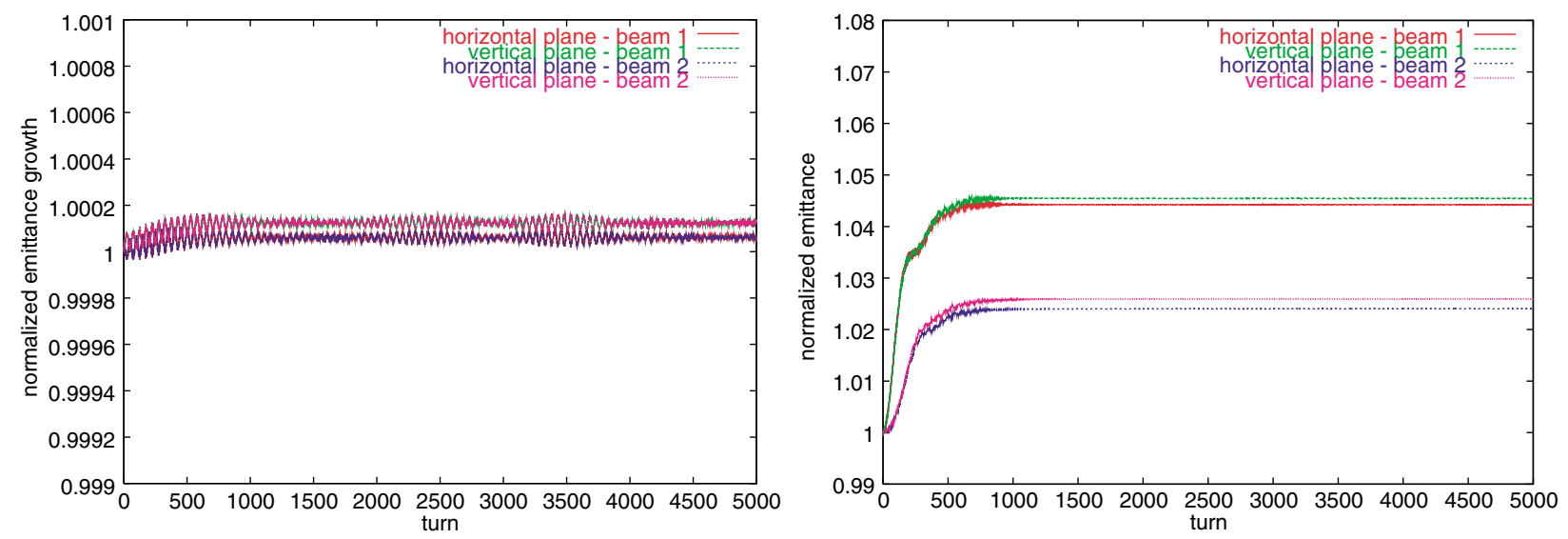

FIG. 7. (Color) The emittance evolution in the strong-strong beam-beam interaction without and with initial $20 \%$ mismatch in both planes of the first beam.
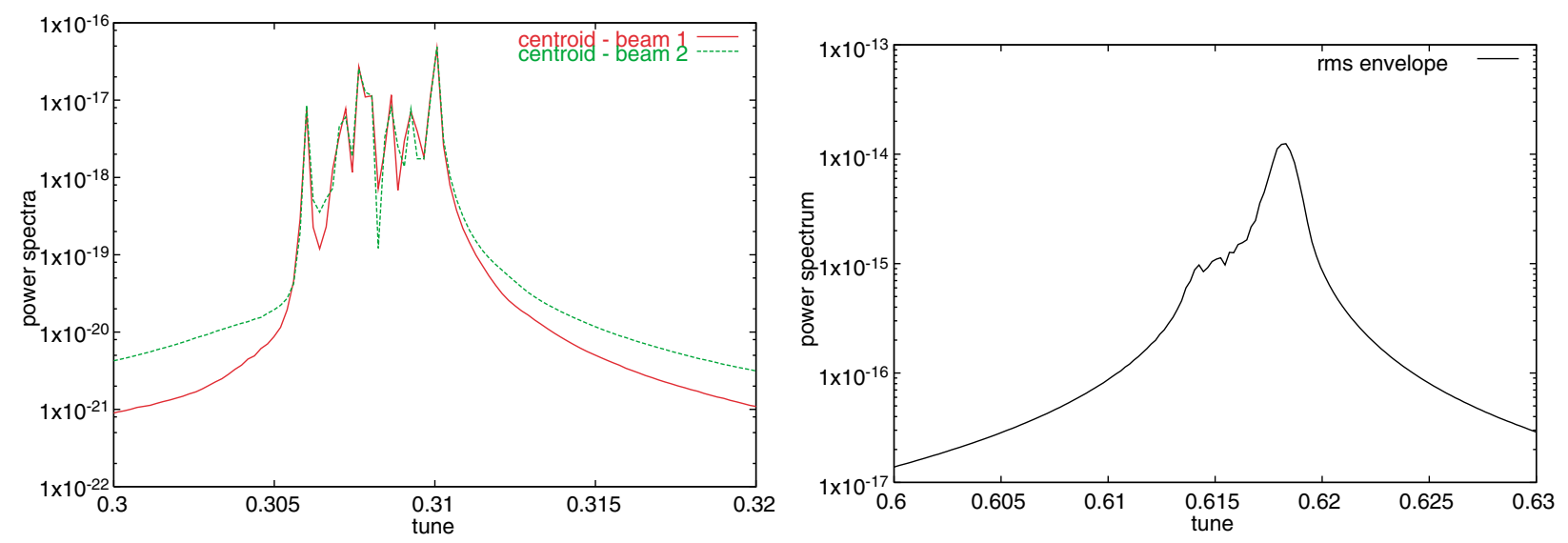

FIG. 8. (Color) The power spectra of the horizontal centroid motion of both beams (left panel) and the power spectrum of the horizontal envelope oscillation of the first beam (right panel).

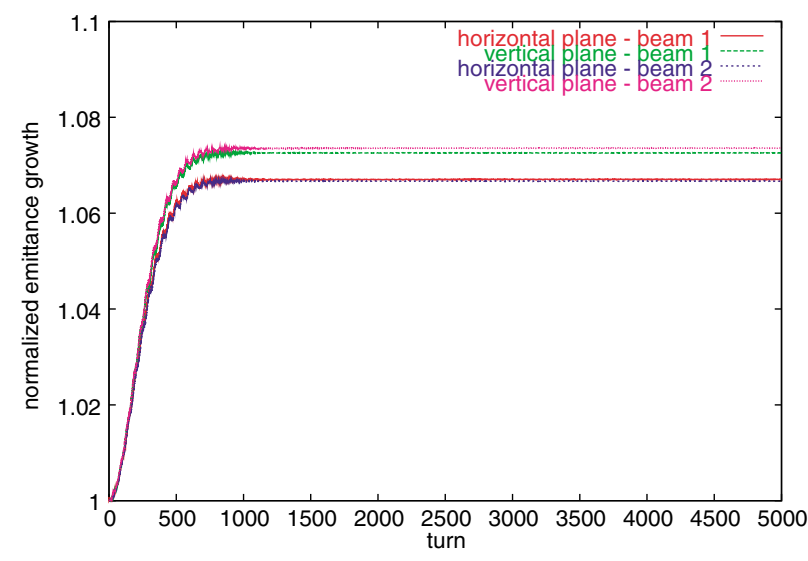

FIG. 9. (Color) The emittance evolution in the strong-strong beam-beam interaction with initial $20 \%$ mismatch in both planes of the two beams. weak-strong beam-beam interaction. However, in the mismatched strong-strong beam-beam interaction, the emittance growth of the mismatched beam cannot be avoided by choosing the machine tune of one beam to be above or below the other beam as in the weak-strong beam-beam interaction. This is due to the fact that in the strong-strong beam-beam interaction, the individual particles within the mismatched beam itself can resonate with the envelope oscillation and cause emittance growth.

\section{CONCLUSIONS}

In this paper, we have studied the halo formation and the emittance growth driven by a parametric resonance from the mismatched beam-beam interaction. In the regime of the weak-strong beam-beam interaction, an onaxis head-on collision between a mismatched strong beam and a weak beam will not cause the formation of 
halo in the weak beam if the two beams have the same machine tunes. When the two beams collide with an offset, a halo is formed in the weak beam due to the $1: 2$ parametric resonance. This causes emittance growth of the weak beam. For two beams with opposite charge, when the weak beam has a lower machine tune than the strong beam, the mismatched strong beam also drives the particles in the weak beam to large amplitude to form a halo. However, when the weak beam has a higher machine tune, there is no halo formation and no significant emittance growth in the weak beam. In the regime of the strong-strong beam-beam interaction, even on-axis headon collisions between two symmetric beams result in emittance growth when even only one beam is mismatched. The emittance growth cannot be avoided by choosing different machine tunes as in the case of the weak-strong beam-beam interaction. This puts a strong requirement for a good beam match during injection in order to avoid emittance growth in colliders if there are no other mechanisms to decohere the envelope oscillation.

\section{ACKNOWLEDGMENTS}

The author thanks Dr. R. Ryne for reading the manuscript. This research used resources of the National Energy Research Scientific Computing Center, which is supported by the Office of Science of the U.S. Department of Energy (US DOE/SC) under Contract No. DE-AC0376SF00098, and the resources of the Center for Computational Sciences at Oak Ridge National Laboratory. Some of the computational work for this project was done on the LBL/NERSC Alvarez Cluster, an 80 node Pentium
III Myrinet cluster. This work was performed under the auspices of a Scientific Discovery through Advanced Computing project, "Advanced Computing for 21st Century Accelerator Science and Technology," which is supported by the US DOE/SC Office of High Energy Physics and the Office of Advanced Scientific Computing Research.

[1] D. Neuffer, A. Riddiford, and A. G. Ruggiero, IEEE Trans. Nucl. Sci. 28, 2494 (1981).

[2] T. Chen, J. Irwin, and R. Siemann, Phys. Rev. E 49, 2323 (1994).

[3] T. Sen and J. A. Ellison, Part. Accel. 55, 293 (1996).

[4] Y. Papaphilippou and F. Zimmermann, Phys. Rev. ST Accel. Beams 5, 074001 (2002).

[5] F. Zimmermann, in Proceedings of Halo'03 ICFA Advanced Beam Dynamics Workshop, 2003, Montauk, NY, AIP Conf. Proc. No. 693 (AIP, New York, 2003).

[6] R. L. Gluckstern, Phys. Rev. Lett. 73, 1247 (1994).

[7] J. Lagniel, Nucl. Instrum. Methods Phys. Res., Sect. A 345, 46 (1994).

[8] C. Chen and R. A. Jameson, Phys. Rev. E 52, 3074 (1995).

[9] R. D. Ryne and S. Habib, Part. Accel. 55, 365 (1996).

[10] T. P. Wangler, K. R. Crandall, R. D. Ryne, and T. S. Wang, Phys. Rev. ST Accel. Beams 1, 084201 (1998).

[11] M. Ikegami, Phys. Rev. E 59, 2330 (1999).

[12] J. Qiang and R. D. Ryne, Phys. Rev. ST Accel. Beams 3, 064201 (2000).

[13] M. A. Furman, Am. J. Phys. 62, 1134 (1994).

[14] J. Qiang, M. A. Furman, and R. D. Ryne, Phys. Rev. ST Accel. Beams 5, 104402 (2002). 